\title{
Aspectos epidemiológicos da seneciose na região sul do Rio Grande do Sul'
}

\author{
Fernando Sérgio Castilhos Karam ${ }^{2}$, Mauro Pereira Soares ${ }^{3}$, Mitsue Haraguchi ${ }^{4}$, \\ Franklin Riet-Correa ${ }^{5}$, Maria del Carmen Méndez $^{6}$ e João André Jarenkow ${ }^{7}$
}

\begin{abstract}
Karam F.S.C., Soares M.P., Haraguchi M., Riet-Correa F., Méndez M.C. \& Jarenkow J.A. 2004. [Epidemiological aspects of seneciosis in southern Rio Grande do Sul, Brazil.] Aspectos epidemiológicos da seneciose na região sul do Rio Grande do Sul, Brasil. Pesquisa Veterinária Brasileira 24(4):191-198. Laboratório de Toxicologia, Faculdade de Medicina Veterinária, URCAMP, Bagé, RS 96400-110, Brazil. E-mail: fernando@alternet.com.br

Seneciosis is the main cause of livestock mortality due to poisonous plants in the State of Rio Grande do Sul, Brazil. This paper presents epidemiological data of 24 outbreaks in cattle and one in horses, diagnosed by the Regional Diagnostic Laboratory at Pelotas University in southern Rio Grande do Sul, from 1998 to 2000 . Additionally, data of 54 outbreaks which occurred in 19781997 were analyzed. The farms where outbreaks occurred in 1998-2000 were visited to obtain clinical and epidemiological data and to verify the presence of Senecio spp. Eleven outbreaks (45.83\%) affected cattle up to 3 years of age, and 13 (54.16\%) cattle over 3 years. Nine (37.5\%) outbreaks affected females and $15(62.5 \%)$ males. Ten (41.66\%) outbreaks occurred in spring, 4 $(16.66 \%)$ in summer, $5(20.83 \%)$ in autumn, and $5(20.83 \%)$ in winter. Morbidity rate was estimated with $4.92 \%$ and case fatality with $95.59 \%$. The predomionating Senecio species were S. brasiliensis on 12 farms (57.14\%), S. selloi on 10 (47.61\%), S. oxyphyllus on 6 (28.57\%), S. heterotrichius on $3(14.28 \%)$, and $S$. leptolobus on 1 farm $(4.76 \%)$. The main clinical signs were progressive emaciation, incoordination, diarrhea, tenesmus, rectal prolapse and aggressiveness. The clinical manifestation periods of affected cattle observed during the farm visits, or of cattle sent for post-mortem examination,were 24-96 hours in 4 outbreaks (16.66\%), 4-7 days in 7 (29.16\%), 1-2 weeks in 4 (16.66\%), 2-3 weeks in 2 (8.33\%), 1-2 months in 2 (8.33\%), and 2-3 months in 1 outbreak (4.16\%). In 4 outbreaks (16.66\%) the clinical manifestation period was not established. In outbreaks with longer clinical manifestation periods some animals showed photosensitization. The main necropsy findings were a hard and enlarged liver, distended gall bladder, edema of the mesenterium and abomasum, and increased amount of liquid in the cavities. Histopathological findings were fibroplasia, megalocytosis and biliary ductal proliferation of the liver, and spongy degeneration of the cerebral white matter. Samples of different Senecio species, in different seasons, were analyzed for detection of pyrrolizidine alkaloids (PAs) by thin layer chromatography. Retrorsine was found in Senecio brasiliensis, S. heterotrichius, S. selloi and S. oxyphyllus. In S. brasiliensis and S. heterotrichius one and two more non-identified PAs were detected, respectively. The total PAs concentration by spectrophotometric method was $0.25 \%$ for $S$. brasiliensis, $0.19 \%$ for $S$. heterotrichius, $0.03 \%$ for $S$.
\end{abstract}

\footnotetext{
${ }^{1}$ Recebido em 25 de maio de 2004.

Aceito para publicação em 21 de junho de 2004.

Parte da dissertação de mestrado do primeiro autor, Pós-Graduação em Patologia Veterinária, Universidade Federal de Pelotas (UFPel).

${ }^{2}$ Laboratório de Toxicologia, Faculdade de Medicina Veterinária, Centro de Ciências Rurais, Universidade da Região da Campanha, Av. Tupy Silveira 2099, Bagé, RS 96400-110. E-mail: fernando@alternet.com.br

${ }^{3}$ Laboratório Regional de Diagnóstico, UFPel, Pelotas, RS 96010-900. E-mail: mpsoares@ufpel.tche.br
}

\footnotetext{
${ }^{4}$ Instituto Biológico de São Paulo, Av. Cons. Rodrigues Alves 1252, São Paulo, SP 04014-002. E-mail: haraguchi@biologico.br

${ }^{5}$ Centro de Saúde e Tecnologia Rural, UFPB, Patos, PB 58700-000. Email: riet@cstr.ufcg.edu.br

${ }^{6}$ Laboratório Regional de Diagnóstico, UFPel, Pelotas, RS 96010-900. E-mail: nane@ufpel.tche.br

7 Depto Botânica, Instituto de Biociências, UFRGS, Av. Bento Gonçalves 9500, Bloco 4, Prédio 43433, Porto Alegre, RS 91501-970. E-mail: jarenkow@portoweb.com.br
} 
oxyphyllus, and $0.03 \%$ for S. selloi. The highest PAs concentration occurred in winter (June/July). No alkaloids were found in samples of S. leptolobus. These results show that $S$. brasiliensis is the most important cause of seneciosis in southern Rio Grande do Sul. Additional data obtained dealt with 54 outbreaks of PAs poisoning in 1978-1997. During this period, 7 outbreaks (12.96\%) affected cattle up to 3 years of age, $39(72.22 \%)$ cattle over 3 years, and 3 outbreaks $(5.55 \%)$ affected cattle of different ages. In 5 outbreaks $(9.25 \%)$ the age was not informed. Seven outbreaks (12.96\%) affected males, $39(72.22 \%)$ females, $3(5.55 \%)$ both sexes, and in 5 outbreaks $(9.25 \%)$ the sex was not informed. Twenty-three outbreaks $(42.59 \%)$ occurred in spring, $9(16.66 \%)$ in summer, $9(16.66 \%)$ in autumn, and $13(24.07 \%$ ) in winter. The greater number of outbreaks during 1998-2000 (24 outbreaks in 3 years) in regard to 1978-1997 (54 outbreaks in 20 years) is probably due to a decrease of more than $50 \%$ in the number of sheep in the region.

INDEX TERMS: Seneciosis, epidemiology, poisonous plants, Senecio spp, Asteraceae, pyrrolizidine alkaloids, cattle.

RESUMO.-Seneciose é a principal intoxicação por planta no sul do Rio Grande do Sul, Brasil. Neste trabalho apresentam-se os dados de 24 surtos de seneciose em bovinos e de um em eqüinos, diagnosticados pelo Laboratório Regional de Diagnóstico (LRD) da Universidade Federal de Pelotas, no período de 1998 a 2000. Adicionalmente são avaliados os dados epidemiológicos de 54 surtos ocorridos no período 1978-1997. Os dados epidemiológicos e clínicos do período 1998-2000 foram coletados em visitas às propriedades onde estavam ocorrendo os surtos. Em $11(45,83 \%)$ surtos os animais tinham até 3 anos de idade e, em 13 (54,16\%), 3 anos ou mais. Quanto ao sexo, 9 $(37,5 \%)$ surtos atingiram fêmeas e 15 (62,5\%) afetaram machos. Em relação à época de ocorrência, $10(41,66 \%)$ surtos ocorreram na primavera, $4(16,66 \%)$ no verão, $5(20,83 \%)$ no outono, e $5(20,83 \%)$ no inverno. A morbidade foi estimada em $4,92 \%$ e a letalidade em 95,59\%. As espécies de Senecio que predominaram nas propriedades visitadas foram $S$. brasiliensis em 12 (57,14\%), $S$. selloi em $10(47,61 \%)$, S. oxyphyllus em $6(28,57 \%)$, S. heterotrichius em $3(14,28 \%)$, e S. leptolobus em $1(4,76 \%)$ propriedade. Os sinais clínicos mais freqüientes foram emagrecimento progressivo, incoordenação, diarréia, tenesmo, prolapso retal e agressividade. O curso clínico da intoxicação, levando em consideração somente os animais necropsiados ou os observados durante as visitas às fazendas, foi de 24 a 96 horas em $4(16,66 \%)$ surtos, de 4 a 7 dias em $7(29,16 \%)$, de 1 a 2 semanas em $4(16,66 \%)$, de 2 a 3 semanas em $2(8,33 \%)$, de 1 a 2 meses em $2(8,33 \%)$, e de 2 a 3 meses em $1(4,16 \%)$ surto. Em quatro $(16,66 \%)$ surtos o período de manifestação clínica não foi observado. Nos surtos em que o curso clínico foi mais prolongado, alguns animais apresentaram fotossensibilização. Os achados de necropsia mais comuns foram fígado aumentado de tamanho e endurecido, vesícula biliar aumentada, edema no mesentério e abomaso e líquido nas cavidades. No fígado, a histopatologia revelou fibroplasia, megalocitose e proliferação de células dos ductos biliares e, no cérebro, degeneração da substância branca. Em amostras das diferentes espécies de Senecio, coletadas em diferentes épocas do ano, foi determinada a concentração de alcalóides pirrolizidínicos (APs) e seus $\mathrm{N}$-óxidos através de cromatografia de camada delgada e espectrofotometria. Retrorsina foi encontrada em Senecio brasiliensis, S. heterotrichius, S. selloi e S. oxyphyllus. Além deste, $S$. brasiliensis e $S$. heterotrichius registraram mais um e dois alcalóides não identificados, respectivamente. A maior con- centração de AP reduzido (AP livre $+N$-óxido) foi encontrada em $S$. brasiliensis no inverno $(0,25 \%)$. Nessa mesma época quantidades decrescentes de AP reduzidos foram encontradas em $S$. heterotrichius $(0,19 \%)$, S. oxyphyllus $(0,03 \%)$ e S. selloi $(0,03 \%)$. Em $S$. leptolobus não foram detectados alcalóides. Esses dados demonstraram que $S$. brasiliensis é a espécie mais importante como causa de seneciose no sul do Rio Grande do Sul. Adicionalmente, foram analisados dados de arquivo do LRD sobre 54 surtos de intoxicação por AP no período 1978-1997. Nesse período, 7 $(12,96 \%)$ surtos afetaram bovinos menores de 3 anos, 39 (72,22\%) afetaram bovinos de 3 anos ou mais, 3 (5,55\%) afetaram bovinos de várias idades e, em $5(9,25 \%)$ surtos a idade não foi informada. Trinta e nove $(72,22 \%)$ surtos afetaram fêmeas, 7 (12,96\%) afetaram machos, 3 (5,55\%) afetaram ambos os sexos e, em 5 (9,25\%), o sexo não foi informado. Vinte e três $(42,59 \%)$ surtos ocorreram na primavera, $9(16,66 \%)$ no verão, $9(16,66 \%)$ no outono, e 13 $(24,07 \%)$ no inverno. O maior número de surtos da intoxicação no período 1998-2000 (24 surtos em 3 anos) em relação ao período 1978-1997 (54 surtos em 20 anos) pode estar relacionado à diminuição em mais de $50 \%$ da população ovina no Estado.

TERMOS DE INDEXAÇÃO: Seneciose, epidemiologia, plantas tóxicas, Senecio spp, Asteraceae, alcalóides pirrolizidínicos, bovinos.

\section{INTRODUÇÃO}

No Rio Grande do Sul, dentre diversas espécies do gênero Senecio, S. brasiliensis, S. oxyphyllus, S. heterotrichius e S. selloi, são frequientemente associadas com casos de intoxicação nos animais, principalmente em bovinos (Méndez et al. 1990, Driemeier et al. 1991, Driemeier \& Barros 1992, Barros et al. 1992, Liddell et al. 1992, Méndez 1993, Méndez \& Riet-Correa 2000). Em uma ocasião a intoxicação foi causada por $S$. tweediei (Méndez \& RietCorrea 1993). A intoxicação por espécies de Senecio em bovinos provoca perdas econômicas importantes pela queda na produtividade dos animais, e mesmo por sua morte, pois os alcalóides pirrolizidínicos (APs), princípios tóxicos da planta, determinam uma lesão irreversível e progressiva no fígado (Bull 1955, Méndez 1993, Pearson 1993, Méndez \& Riet-Correa 2000). Estima-se que no Rio Grande do Sul, Estado que conta com uma população bovina de 13 milhões de cabeças, 5\% dessa população morre anualmente, equivalendo a 650.000 animais por ano. Desses, $10 \%$ a $14 \%$ morrem devido a intoxicações por plantas, o que 
corresponde de 64 mil a 90 mil cabeças/ano. Estimando um preço médio de US\$ 200 por animal, apenas as perdas diretas por mortes atribuídas a plantas tóxicas, no Rio Grande do Sul, ficam entre US\$12 a 18 milhões anualmente. Aproximadamente $50 \%$ das mortes causadas por plantas tóxicas devem-se à intoxicação por diversas espécies de Senecio, concluindo-se, portanto, que esta intoxicação ocasiona perdas econômicas de, em média, US\$ 7,5 milhões anuais (Riet-Correa \& Medeiros 2001, Schild et al. 2004). Os resultados de um estudo fenológico foram publicados anteriormente (Karam et al. 2002).

O objetivo principal desse trabalho foi estudar alguns fatores epidemiológicos da seneciose e/ou suas inter-relações, assim como relacionar a ocorrência da intoxicação animal com a presença de alcalóides pirrolizidínicos.

\section{MATERIAL E MÉTODOS}

Este estudo baseou-se em visitas às propriedades para acompanhamento in loco de todos os surtos registrados no Laboratório Regional de Diagnóstico (LRD) da Faculdade de Veterinária, da Universidade Federal de Pelotas (UFPel), de janeiro de 1998 a dezembro de 2000. Em cada surto foram registrados a localização das propriedades (municípios), a idade e sexo dos animais atingidos, o curso clínico da doença e a presença e época de ocorrência de espécies de Senecio. A análise dos componentes epidemiológicos foi feita em dados percentuais, sem correlação, apenas considerando Senecio spp como agente, bovinos como hospedeiros e a propriedade rural como meio ambiente.

Foram analisados, também, dados de arquivo do LRD de agosto de 1978 (ano de criação do LRD) a dezembro de 1997, num estudo retrospectivo para avaliação da intoxicação por APs no período anterior ao presente trabalho.

Para relacionar o número de ocorrências da seneciose em bovinos, com a evolução da criação ovina no Rio Grande do Sul, ao longo do período estudado, foram coletados dados referentes a essa população em municípios da região sul do Estado, onde a criação de ovinos é mais expressiva.

A determinação dos APs e dos seus teores nas espécies de Senecio envolvidas com a intoxicação nesse período foi realizada coletandose amostras de folhas das plantas em diferentes épocas do ano nos municípios de Bagé, RS (S. brasiliensis e $S$. oxyphyllus) e Capão do Leão, RS (S. brasiliensis, S. heterotrichius, S. selloi e S. leptolobus), em regiões onde ocorreram surtos. A detecção dos alcalóides foi obtida através da técnica de cromatografia de camada delgada (CCD). Cada amostra das folhas secas foi extraída com etanol a 96\% por maceração e em seguida o extrato foi concentrado até a secura. Este resíduo etanólico, após acidificação e filtração, foi alcalinizado até pH 9 para em seguida ser extraído em clorofórmio. A solução clorofórmica foi concentrada totalmente e uma alíquota de cada amostra foi aplicada em placas de vidro impregnadas de sílica-gel 60G e desenvolvida no sistema de solventes constituídos de clorofórmio-metanol-hidróxido de amônio 17\% (82,5:15,5:2), seguida de revelação inicial em solução de orto-cloranil e em seguida com solução de Ehrlich, conforme Molyneux \& Roitman (1980), tendo como amostra padrão, alcalóide retrorsina (Sigma). A análise espectrofotométrica dos alcalóides pirrolizidínicos (AP livre), seus $\mathrm{N}$-óxidos e dos AP reduzidos (AP livre + AP $N$-óxido) foi determinada conforme Mattocks $(1968$ a,b) nas folhas secas de $S$. brasiliensis, S. heterotrichius, S. selloi, S. oxyphyllus e $S$. leptolobus. A curva de calibração foi determinada através do retrorsina padrão (Sigma) na faixa entre 5 e $30 \mathrm{mg}$, sendo realizada a leitura de absorbância em 560 nm utilizando espectrofotômetro modelo U-2001 da marca Shimadzu.

\section{RESULTADOS}

\section{Surtos no período $1998-2000$}

De janeiro de 1998 a dezembro de 2000 registraram-se 24 surtos de seneciose em bovinos, assim distribuídos nos seguintes municípios da região sul do Rio Grande do Sul: Arroio Grande, 5 surtos; Bagé, 2; Canguçu, 2; Capão do Leão, 3; Cerrito, 2; Herval, 1; Jaguarão, 2; Pedro Osório, 1; Pelotas, 1; Pinheiro Machado, 1; Piratini, 3; e Retiro, 1 surto. Em um dos surtos de Capão do Leão os animais acometidos eram oriundos de Piratini e Santa Vitória. Em um dos surtos observados em Bagé os animais eram oriundos de Cerrito e, em um dos surtos de Jaguarão eram provenientes de Arroio Grande.

Um caso registrado em Canguçu afetou um eqüino, fêmea, de 23 anos, na primavera de 1999. Cerca de 15 dias após parir, a égua apresentou emagrecimento progressivo, incoordenação, anorexia e temperatura de $40-41^{\circ} \mathrm{C}$, durante 2 a 3 semanas, e morreu. Nessa propriedade predominavam Senecio oxyphyllus e S. selloi, num campo que indicava grande lotação de animais.

Os dados referentes a sexo, idade dos animais afetados e época do ano na qual ocorreram os surtos apresentam-se no Quadro 1; adicionalmente são incluídos os dados referentes ao período 1978-1997. No período 1998-2000, a morbidade do rebanho exposto à ingestão de Senecio spp foi estimada em $4,92 \%$ e a letalidade foi de $95,59 \%$.

Quadro 1. Seneciose em bovinos no sul do Rio Grande do Sul, no período $1978-2000$

\begin{tabular}{lccc}
\hline Período analisado & $\begin{array}{c}1978-1997 \\
\mathrm{~N}^{\circ}(\%)\end{array}$ & $\begin{array}{c}1998-2000 \\
\mathrm{~N}^{\circ}(\%)\end{array}$ & $\begin{array}{c}\text { Total } \\
\mathrm{N}^{\circ}(\%)\end{array}$ \\
\hline Idade & & & \\
$\quad$ Menores de 3 anos & $7(12,96)$ & $11(45,83)$ & $18(23,07)$ \\
3 anos ou mais & $39(72,22)$ & $13(54,16)$ & $52(66,66)$ \\
$\quad$ Várias & $3(5,55)$ & 0 & $3(3,84)$ \\
$\quad$ Não informado & $5(9,25)$ & 0 & $5(6,41)$ \\
Sexo & $7(12,96)$ & $15(62,5)$ & $22(28,20)$ \\
$\quad$ Machos & $39(72,22)$ & $9(37,5)$ & $48(61,53)$ \\
$\quad$ Fêmeas & $3(5,55)$ & 0 & $3(3,84)$ \\
$\quad$ Ambos sexos & $5(9,25)$ & 0 & $5(6,41)$ \\
Não informado & $9(16,66)$ & $4(16,66)$ & $13(16,66)$ \\
Época do ano & $9(16,66)$ & $5(20,83)$ & $14(17,94)$ \\
$\quad$ Verão & $13(24,07)$ & $5(20,83)$ & $18(23,07)$ \\
$\quad$ Outono & $23(42,59)$ & $10(41,66)$ & $33(42,31)$ \\
Inverno & 54 & 24 & 78 \\
$\quad$ Primavera & 54 & &
\end{tabular}

A duração do curso clínico, levando em consideração somente os animais necropsiados ou os observados durante as visitas às fazendas, foi a seguinte: em $4(16,66 \%)$ surtos os animais manifestaram a intoxicação num período de 24 a 96 horas, em $7(29,16 \%)$ de 4 a 7 dias, em $4(16,66 \%)$ de 1 a 2 semanas, em $2(8,33 \%)$ de 2 a 3 semanas, em $2(8,33 \%)$ de 1 a 2 meses, e em 1 $(4,16 \%)$ surto de 2 a 3 meses. Em $4(16,66 \%)$ surtos o período de manifestação clínica não foi observado; num deles, um único animal acometido apresentava apenas diarréia há algum tempo e foi feita biopsia hepática, diagnosticando-se lesões compatíveis com intoxicação por APs, tais como fibroplasia e presença de poucos hepatócitos. Na visita a essa propriedade detectou-se 
presença de Senecio spp, com predomínio de S. selloi, mas o animal acometido já tinha sido comercializado e não se obteve maiores informações. Num dos surtos de Capão do Leão, na visita para coleta de dados, o proprietário já havia trocado de ramo produtor e não se conseguiu maiores informações. No surto ocorrido em Retiro obteve-se apenas a informação de que as mortes tinham sido súbitas, sem observação de sinais clínicos. Um outro surto ocorrido no município de Bagé, numa propriedade livre de Senecio spp, animais aparentemente sadios oriundos do município de Cerrito, de uma propriedade onde havia a planta e tinha se registrado a doença, manifestaram sinais da intoxicação e morte entre 1 e 2 meses depois da compra e transporte dos mesmos, mas sem informações sobre a duração do curso clínico nessa propriedade.

Em um surto no município de Jaguarão, os animais afetados que se encontravam em área com pouco Senecio, mas também com pouca oferta de pasto, eram oriundos de um estabelecimento no município de Arroio Grande e tinham sido transportados há cerca de 2 a 3 semanas quando, então, começaram a manifestar os sinais clínicos na propriedade de Jaguarão. Em visita à propriedade de origem desses animais observou-se quantidade expressiva de $S$. brasiliensis e $S$. selloi, além de pouca disponibilidade de pastagens, o que parecia indicar superlotação animal.

Os sinais clínicos mais freqüientes foram emagrecimento progressivo, incoordenação, diarréia intermitente, tenesmo, prolapso de reto e agressividade. Outros sinais observados com frequiência variável foram: fezes endurecidas e escuras, sialorréia, hipertermia, hipotermia, apatia, decúbito, tremores musculares, andar em círculo e pêlo arrepiado e quebradiço. Fotossensibilização foi observada em $9(37,5 \%)$ desses surtos, mas nem todos animais afetados apresentaram esse sinal. As lesões se manifestaram basicamente por descamação nas orelhas, focinho, dorso, e úlceras na face inferior da língua. Em muitos desses animais, segundo o histórico mencionado pelos produtores, a fotossensibilização foi passageira e se manifestou naqueles que tiveram um curso clínico de, no mínimo, uma semana. Num surto ocorrido em Piratini, uma vaca apresentou um curso clínico de 6 meses até a morte e, nesse período, teve fotossensibilização e se recuperou desse sintoma. Num outro surto, em Arroio Grande, uma vaca apresentou fotodermatite e, recuperada desse sintoma, permanecia viva, mas apática, um ano após o primeiro registro de seneciose nessa propriedade.

Os achados de necropsia mais freqüentes foram hepatomegalia, fígado endurecido e pálido, vesícula biliar com parede espessada e dilatada pelo acúmulo de bile, petéquias endocárdicas e pericárdicas, mobilização de gorduras, edema principalmente no mesentério e abomaso, e aumento de líquido nas cavidades.

As principais lesões histológicas observadas foram megalocitose, proliferação de células dos ductos biliares e fibrose difusa, no fígado. Alguns casos apresentaram hepatócitos com vacúolos citoplasmáticos, hemorragias multifocais, nódulos regenerativos, infiltração de células inflamatórias, espessamento da cápsula de Glisson ou necrose centrolobular. No cérebro observou-se degeneração esponjosa da substância branca, especialmente na substância branca sub-cortical e cápsula interna. De acordo com a observação visual, as espécies de Senecio consideradas como predominantes em 21 propriedades visitadas, das 24 onde ocorreram os surtos de intoxicação nesse período, tiveram a seguinte distribuição por propriedade: $S$. brasiliensis foi predominante em $12(57,14 \%)$ propriedades, S. selloi em $10(47,61 \%)$, S. oxyphyllus em $6(28,57 \%)$, S. heterotrichius em $3(14,28 \%)$, e S. leptolobus em 1 $(4,76 \%)$ propriedade. Em várias propriedades duas ou mais espécies de Senecio apresentavam-se, aparentemente, numa população equilibrada, daí considerava-se o predomínio de ambas ou mais.

Nas propriedades observou-se, em geral, quantidade considerável de Senecio spp e sempre pouca oferta de pasto nativo, ou pastagem, de boa qualidade, em relação ao número de animais no estabelecimento e, ainda, que as plantas de Senecio encontravam-se em diferentes estádios vegetativos e com bom vigor. Além da aparente sobrecarga animal e baixa cobertura de pastagens observaram-se, em uma propriedade, várias plantas de S. selloi, adultas, com evidências de terem sido consumidas em sua porção superior, de floração.

Em apenas uma das propriedades com seneciose havia ovinos.

Na pesquisa de alcalóides, as amostras de $S$. brasiliensis, $S$. heterotrichius, S. selloi e S. oxyphyllus registraram manchas de coloração magenta em $R_{f} 0,50$, idêntica com o padrão de retrorsina, através de CCD quando revelada com reagente de Ehrlich. Por seu lado, as amostras de $S$. brasiliensis apresentaram mais uma mancha $\left(\mathrm{R}_{\mathrm{f}} 0,57\right)$ e as de $S$. heterotrichius mais duas $\left(\mathrm{R}_{\mathrm{f}} 0,53\right.$ e 0,70$)$, indicando a presença de mais um e dois alcalóides, respectivamente. Nas folhas de $S$. leptolobus não foi detectada a presença de APs nas mesmas condições analisadas acima. Pelas análises espectrofotométricas (Quadro 2) foi registrada maior concentração de APs reduzido (AP livre $+N$-óxidos) em $S$. brasiliensis no inverno $(0,25 \%)$ quando a planta estava em brotação. Nessa mesma época, os teores de APs reduzidos foram $0,19 \% \mathrm{em} S$. heterotrichius, $0,03 \%$ em S. oxyphyllus e $0,03 \%$ em S. selloi. A amostra de S. leptolobus não acusou a presença de APs, portanto, não foi executada análise espectrofotométrica para dosagem do mesmo.

Quadro 2. Variação sazonal do teor de alcalóides pirrolizidínicos (AP) em Senecio spp que causaram surtos de intoxicação

\begin{tabular}{lcccc}
\hline Espécie & $\begin{array}{c}\text { Coleta } \\
\text { Mês/Ano }\end{array}$ & $\begin{array}{c}\text { AP } \\
\text { Livre g \% }\end{array}$ & $\begin{array}{c}\text { AP } \\
\text { N-óxido g \% }\end{array}$ & $\begin{array}{c}\text { AP } \\
\text { Totais g \% }\end{array}$ \\
\hline \multirow{2}{*}{ S. brasiliensis } & Julho/1998 & 0,151 & 0,100 & 0,251 \\
& Junho/1999 & 0,200 & 0,006 & 0,206 \\
& Setembro/1999 & 0,133 & 0,088 & 0,221 \\
& Novembro/1999 & 0,082 & 0,096 & 0,178 \\
S. heterotrichius & Julho/1998 & 0,070 & 0,120 & 0,190 \\
& Junho/1999 & 0,041 & 0,093 & 0,134 \\
& Setembro/1999 & 0,014 & 0,076 & 0,090 \\
S. selloi & Novembro/1999 & 0,058 & 0,087 & 0,015 \\
& Julho/1998 & 0,012 & 0,009 & 0,021 \\
& Junho/1999 & 0,019 & 0,007 & 0,026 \\
Outubro/1999 & 0,005 & 0,003 & 0,008 \\
& Novembro/1999 & 0,006 & 0,010 & 0,016 \\
& Junho/1999 & 0,016 & 0,012 & 0,028 \\
& Setembro/1999 & 0,011 & 0,044 & 0,055 \\
& Novembro/1999 & 0,003 & 0,022 & 0,025 \\
& Janeiro/2000 & 0,003 & 0,193 & 0,196
\end{tabular}




\begin{tabular}{|c|c|c|c|}
\hline Município & $\begin{array}{c}\text { População/Ano } \\
1963 \text { a }\end{array}$ & $1996^{\mathrm{b}}$ & $2000^{c}$ \\
\hline Bagé & $\begin{array}{c}939.200 \\
-\end{array}$ & $\begin{array}{l}311.552 \\
25.507^{d} \\
18.293^{d}\end{array}$ & $\begin{array}{c}145.390 \\
27.371^{\mathrm{d}} \\
15.844^{\mathrm{d}}\end{array}$ \\
\hline Caçapava do Sul & 152.000 & 87.988 & 80.307 \\
\hline Dom Pedrito & 652.000 & 236.643 & 189.260 \\
\hline Herval do Sul & 378. & 212.427 & $-\mathrm{e}$ \\
\hline Lavras do Sul & 234.000 & 91.2 & 103.072 \\
\hline Pinheiro Machado & 342.000 & 164.462 & 123.022 \\
\hline Piratini & 274.000 & 120.000 & $-\mathrm{e}$ \\
\hline Santana do Livramento & 910.200 & 564.250 & - e \\
\hline \multicolumn{4}{|c|}{$\begin{array}{l}\text { a Fonte: Nunes Vieira } 1967 . \\
\text { b Fonte: Secretaria Municipal de Agropecuária do município de Bagé. } \\
\text { c Fonte: Inspetoria Veterinária e Zootécnica de Bagé, DPA/SAA, RS. } \\
\text { d Candiota e Hulha Negra, respectivamente, municípios emancipados de } \\
\text { Bagé. } \\
\text { e Dados não obtidos. }\end{array}$} \\
\hline
\end{tabular}

\section{Surtos registrados no período 1978-1997}

Na área de abrangência do LRD, no período de agosto de 1978 a dezembro de 1997 foram registrados 54 surtos de intoxicação por APs, em bovinos (Quadro 1).

Nesse período de 1978 a 1997, os sinais clínicos relatados são similares aos já descritos, acrescidos, em alguns casos, de icterícia e fezes ressequidas com estrias de sangue. Fotossensibilização ocorreu em $8(14,81 \%)$ surtos, sendo o principal sinal em 4 deles. Esses surtos com fotossensibilização ocorreram em bovinos com curso clínico de 10 dias até 6 meses. Aos achados de necropsia observados no período 1998-2000 acrescentamse nódulos na vesícula biliar e fígado com aspecto de noz moscada em alguns casos.

\section{Evolução da população de ovinos no período 1963-2000}

O Quadro 3 apresenta a população ovina no Rio Grande do Sul, em municípios onde essa criação é mais expressiva. Observa-se, nesta região, ao longo dos anos, o declínio acentuado da ovinocultura.

\section{DISCUSSÃO E CONCLUSÕES}

A intoxicação por Senecio spp em bovinos é a segunda causa de morte de bovinos no Rio Grande do Sul, responsável por aproximadamente $7 \%$ de todas as mortes, sendo superada somente pela tristeza parasitária (Riet-Correa \& Medeiros 2001). Além das perdas ocasionadas pela morte dos animais, as perdas econômicas incluem as ocasionadas pelas tentativas ineficazes de tratamento, o gasto com mão de obra, e a provável ocorrência de casos subclínicos. Além disso, em termos de número de ocorrências, a extensão do problema é bem maior do que aquele que chega ao laboratório de diagnóstico (Driemeier et al. 1991, Barros et al. 1992). Por outro lado, o aumento no número anual de surtos no período 1998-2000 (24 surtos em 3 anos) em relação ao período 1978-1997 (54 surtos em 20 anos) sugere que as perdas por mortes foram mais importantes no período mais recente e, portanto, a frequiência e a importância econômica da intoxicação aumentou no período 1998-2000. Esse aumento na freqüência da intoxicação pode estar relacionado à diminuição da população ovina no Estado (Quadro 3) e, conseqüentemente, ao aumento da oferta de Senecio spp. Embora os ovinos possam adoecer espontaneamente (Ilha et al. 2001), a intoxicação não é comum nessa espécie, por ser mais resistente à ação dos alcalóides (Radostits et al. 1999) e, por isso, podem ser usados como controladores naturais da planta (Méndez \& Riet-Correa 2000, Tokarnia et al. 2000), mas há que se considerar a possibilidade da dispersão de sementes através do estrume dessa espécie animal (Harper \& Wood 1957). Na Grã-Bretanha, um dos fatores considerado importante para o aumento da população de Senecio jacobaea foi a diminuição da criação de ovinos (Harper \& Wood 1957). No Rio Grande do Sul surtos da intoxicação em bovinos são relatados em propriedades onde não há criação de ovinos (Barros et al. 1987, 1992, Méndez et al. 1987, Driemeier et al. 1991). Em um trabalho anterior foi constatado que uma lotação de ovinos igual ou maior que 0,43 animais por hectare, em pastoreio contínuo, controlam a ocorrência de Senecio spp (Soares et al. 2000).

No período abordado houve registro de apenas um caso em eqüinos. A ocorrência da seneciose é rara em eqüinos no Sul do Brasil. Isto se deve, provavelmente, a que essa espécie animal costuma receber suplementação com grãos ou concentrados na época de carência de forragem verde, justamente a de maior risco para a ingestão de Senecio spp, além do hábito de pastoreio mais seletivo desses animais (Gava \& Barros 1997). Por outro lado deve ser considerado que a população de eqüinos (aprox. 300 mil) no Estado é muito menor que a população de bovinos (aprox. 13 milhões).

No período 1978-1997, a doença foi mais freqüente em fêmeas do que em machos; no entanto, durante o período 1998-2000 os machos foram mais afetados. A observação de que as vacas são a categoria mais afetada deve-se, provavelmente, ao fato de que, por serem animais que permanecem por períodos maiores na propriedade ingerem, ao longo do tempo, maior quantidade de Senecio (Méndez \& Riet-Correa 2000). Por outro lado, os machos são mais suscetíveis, entretanto, por permanecerem menor tempo na propriedade, são menos afetados. A maior susceptibilidade dos machos em relação às fêmeas pode dever-se, em parte, ao tipo de lesão provocada pelos APs, cuja metabolização inicial, no fígado, se dá através da ativação do sistema citocromo P-450. As enzimas pertencentes a esse sistema têm mais atividade nos machos do que nas fêmeas e variam conforme a idade, pois em recém-nascidos têm menor atividade que nos adultos (MacLachlan \& Cullen 1998). Para Pearson (1993) os bezerros são muito mais sensíveis que os adultos, mas neste estudo observou-se que a maioria dos animais afetados tinha 3 ou mais anos de idade, caracterizando-a como doença de adultos, independentemente do sexo do animal. O desenvolvimento das lesões ocorre gradativamente e os sinais clínicos não ocorrem até haver suficiente lesão hepática para alterar a função, quando, então, aparecem de forma quase sempre súbita e, com frequiência, algum tempo após os animais terem parado de ingerir o material tóxico (Kingsbury 1964, Radostits et al. 1999). 
Os sinais clínicos observados, assim como os achados macroscópicos e microscópicos coincidem com o descrito por diversos autores (Summers et al. 1995, Méndez \& Riet-Correa 2000, Tokarnia et al. 2000). A fotossensibilização devido à intoxicação por Senecio spp ocorre naqueles animais com período clínico mais prolongado (Schild et al. 2004). A ocorrência desse sinal está ligada ao grau de comprometimento hepático. As lesões provocadas pela metabolização dos APs, impedindo a mitose dos hepatócitos, com a consequiente degeneração e morte destes, ocasionam uma fibrose inicialmente periportal, que pode evoluir atingindo a região centrolobular, tornando-se difusa. A fibrose pode determinar colestase intra-hepática e retenção e acúmulo de bile no sangue periférico, com presença do agente fotodinâmico filoeritrina e a ocorrência da fotossensibilização secundária ao dano hepático (Driemeier et al. 1991, Jubb et al. 1992, Méndez 1993, Pearson 1993, MacLachlan \& Cullen 1998).

Quanto à época de ocorrência da doença, primavera e inverno são as estações com maior número de surtos. A maior freqüiência da intoxicação nessas épocas revela, aparentemente, o maior consumo de Senecio durante fim de outono e inverno, geralmente épocas de escassez de pasto de boa qualidade (Méndez 1993). Uma vez que a lesão desenvolve-se lentamente (Bull 1955), é provável que os animais que tenham ingerido a planta durante fim de outono e inverno adoeçam no final de inverno e primavera. Isto ficou evidenciado nos dois surtos que ocorreram 2 a 4 semanas após o transporte de bovinos de áreas onde havia Senecio spp para áreas onde a planta não ocorria ou encontrava-se em pouca quantidade. As condições ambientais de pouca oferta de pasto, superlotação bovina e presença de Senecio spp. no inverno são favoráveis à ingestão e, se as plantas novas estão estreitamente associadas ao capim, o perigo de ingestão pelos bovinos é ainda maior (Tokarnia \& Döbereiner 1984, Schild et al. 1989, Driemeier et al. 1991, Driemeier \& Barros 1992, Tokarnia et al. 2000). Além disso, o outono e o inverno são as épocas mais propícias à emergência e ao estabelecimento de plântulas (Castellani et al. 1999) e, às vezes, se constituem na única oferta de plantas verdes aos animais durante o período outono-inverno. Outro fator que evidentemente colabora para a ocorrência da intoxicação no inverno e primavera é que no período de escassez (junho/julho) é maior o teor de alcalóides nas espécies de Senecio da região estudada (Quadro 2). O maior número de surtos na primavera pode ser devido, também, em parte, a que é nessa estação que ocorrem grandes mudanças de manejo nas propriedades, visando principalmente à reprodução. Além de ser a época tradicional de parição, são mais freqüentes as feiras, vendas e transporte de animais. A demanda fisiológica provocada por essas situações estressantes é agravada naqueles animais que ingeriram APs numa estação anterior, terminando por manifestarem a intoxicação latente. Em algumas ocasiões, o aparecimento dos sinais clínicos parece estar associado a essas situações e, na ausência de uma maior exigência do organismo, o dano hepático pode não se manifestar (Dickinson 1980, Peterson \& Culvenor 1983, Johnson \& Smart 1983, Barros et al. 1992, Méndez 1993). Um fator que pode favorecer a ocorrência da intoxicação no inverno é a maior demanda metabólica nessa época do ano.

Apesar da maior frequiência da intoxicação durante o inver- no e primavera, numerosos surtos foram observados, também, no verão e outono (Quadro 1). Esse fato pode ser devido a dois fatores: um período mais prolongado desde a ingestão até o aparecimento dos sinais clínicos; e a ingestão da planta nas diversas épocas do ano. A presença de Senecio spp, em diferentes fases de crescimento, em diferentes épocas do ano, foi observada na maioria das propriedades com casos da intoxicação. As fases das plantas são influenciadas pela precipitação, umidade do solo, luz e temperatura, e pelo manejo das pastagens em cada propriedade. No entanto, os fatores ambientais para a permanência de plantas indesejáveis, como Senecio spp, não são tão importantes se estas já estão estabelecidas no ambiente, mantendo-se verdes durante o período outono-inverno ou em outras épocas em que a condição ambiental seja similar. Se as condições ambientais são favoráveis à emergência, o seu desenvolvimento pode ocorrer em qualquer época do ano e, conseqüentemente, a ingestão e a intoxicação em diferentes épocas. $\mathrm{Na}$ região estudada observa-se que sempre há Senecio spp em estádio vegetativo, o que indica constante disponibilidade aos animais (Karam et al. 2002).

Neste estudo a maior concentração de alcalóides ocorreu na fase de brotação de Senecio spp. (Quadro 2). Resultados semelhantes são mencionados por Méndez (1993). No inverno ocorre, também, a maior ingestão da planta, mas bovinos muito famintos podem ingerir a planta adulta (Tokarnia et al. 2000). A dose tóxica é bastante variável de acordo com a espécie e seu estádio de crescimento, o teor de alcalóides presentes, o período de ingestão (Méndez et al. 1990, Tokarnia et al. 2000) e os fatores relacionados ao próprio animal (Martin et al. 1988, MacLachlan \& Cullen 1998). Para Hirschmann et al. (1987), Krebs et al. (1996), Osweiler (1998) e Radostits et al. (1999), o estádio de floração é considerado o mais rico em alcalóides. As condições de meio ambiente, como seca e altas temperaturas, parecem aumentar o teor de APs presentes nas plantas e, portanto, determinada espécie de Senecio pode ser mais tóxica dependendo do meio ambiente e suas variáveis (Radostits et al. 1999).

A análise por método espectrofotométrico revelou que os alcalóides pirrolizidínicos (APs livres) e seus $\mathrm{N}$-óxidos, de modo geral, apresentaram teores de APs totais mais elevados em $S$. brasiliensis, seguido de $S$. heterotrichius e diminuindo drasticamente em S. oxyphyllus e S. selloi. Em S. heterotrichius e S. oxyphyllus os $\mathrm{N}$-óxidos mostraram-se aumentados relativos às bases livres. Esses resultados são semelhantes aos encontrados por Méndez et al. (1990) que determinaram 0,31\% de APs em S. brasiliensis, $0,19 \%$ em S. heterotrichius, e $0,10 \%$ em S. selloi.

Senecio brasiliensis foi, também, uma das duas espécies mais freqüentes e predominantes nas propriedades com surtos da intoxicação, o que confirma, portanto, que esta é a espécie mais importante na ocorrência da seneciose na região estudada. $\mathrm{Na}$ análise da variação sazonal dos APs das espécies de Senecio acompanhadas observa-se que a época de maior teor de alcalóides (junho/julho) coincide com o outono/inverno (Quadro 2), justamente quando as fases vegetativas são mais vigorosas. $O$ fato de S. oxyphyllus, na coleta de janeiro de 2000 , ter valores próximos a $S$. brasiliensis em outras estações deve estar relacionado diretamente às condições de ambiente, em especial à precipitação. No local de coleta de $S$. oxyphyllus choveu cerca de $1000 \mathrm{~mm}$ a me- 
nos que no ano anterior, o que foi determinante na população observada a ponto de nenhum exemplar sobreviver a esse período de baixa precipitação. Apenas o local de coleta, uma região mais baixa em relação às demais, manteve uma certa umidade $e$ assim que começaram as precipitações, as plantas emitiram muitos brotos vegetativos, tornando-se mais vigorosas, similar ao comportamento de outono/inverno (Karam et al. 2002).

Senecio leptolobus é, também, potencialmente tóxica aos bovinos (Méndez \& Riet-Correa 2000). Neste estudo registrou-se sua ocorrência em 3 propriedades, mas era dominante em apenas uma delas, onde também havia S. selloi na mesma proporção. A amostra de S. leptolobus para análise do teor de APs não acusou presença do princípio ativo, o que confirma, nessas condições, $S$. leptolobus como espécie não envolvida na epidemiologia da seneciose. Resultados semelhantes foram obtidos por Méndez et al. (1990) que encontraram baixa concentração de PAs $(0,005 \%)$ nesta espécie, que foi a única que não resultou tóxica para frangos.

A ocorrência de um número maior de surtos de seneciose no Rio Grande do Sul, em determinados anos, foi precedido por severa estiagem e alta infestação de Senecio nos campos (Driemeier et al. 1991). Às vezes, os fatores de meio ambiente, como frio excessivo, ocorrência de geadas e o pisoteio de animais numa área superpovoada são determinantes para o estabelecimento de plantas indesejáveis, uma vez que a intensidade de pastoreio influi na evolução da vegetação campestre (Gonçalves \& GirardiDeiro 1986, Gonçalves et al. 1988). Por outro lado, devemos considerar a sazonalidade do campo natural, que provoca alterações na fisionomia dos campos da região Sudoeste do Estado, determinada por déficit hídrico e por baixas temperaturas nos meses de inverno (Girardi-Deiro \& Gonçalves 1985, Girardi-Deiro et al. 1992). Um pastoreio pesado no inverno, ou em época de alta precipitação, contribui para a diminuição da camada de cobertura vegetal, aumentando a incidência de luz no solo e, conseqüentemente, a temperatura, o que favorece a germinação de sementes ali presentes (Thompson et al. 1977, Coombs et al. 1991, Beskow 1995). Esses fatores ambientais, especialmente da baixa cobertura vegetal em relação à lotação animal foram observados em algumas propriedades onde ocorreram surtos da doença. Além disso, McClements et al. (1998) observaram que solos mais ricos em fósforo têm menor infestação de Senecio jacobaea e que um baixo $\mathrm{pH}$ do solo parece favorecer sua incidência. Esses fatores são comuns em várias regiões do Rio Grande do Sul (Macedo 1984), incluindo solos da região (Bagé e Capão do Leão) acompanhada neste trabalho.

Há uma relação entre os aspectos epidemiológicos e eventos fenológicos. $\mathrm{O}$ componente meio ambiente e o agente Senecio influem diretamente sobre o componente hospedeiro, podendo causar um desequilíbrio, ou seja, a doença, caracterizando a estrutura epidemiológica (Martin et al. 1988, Rouquayrol 1990). A ocorrência do agente Senecio, as condições ambientais para a existência do mesmo, especialmente a umidade e a luz para a emergência e brotação, e a temperatura para a floração, são variáveis que contribuem para o estabelecimento e permanência do agente no meio ambiente e, os animais suscetíveis à intoxicação, dependendo da oferta de pasto, densidade populacional e de variáveis individuais, ingerem o agente tóxico e podem desenvolver a seneciose. Tais aspectos epidemiológicos interatuantes devem ser analisados e conhecidos para quantificar-se a importância de cada um na expressão dessa toxicose e para tomar as medidas profiláticas adequadas.

Agradecimentos.- Trabalho apoiado financeiramente pelo Programa de Apoio a Núcleos de Excelência (PRONEX), Processo ${ }^{\circ} 7697102600$ e pela Fundação de Apoio à Pesquisa do Rio Grande do Sul (FAPERGS).

\section{REFERÊNCIAS}

Barros C.S.L., Metzdorf L.L. \& Peixoto P.V. 1987. Ocorrência de surtos de intoxicação por Senecio spp. (Compositae) em bovinos no Rio Grande do Sul. Pesq. Vet. Bras. 7(4):101-107.

Barros C.S., Driemeier D., Pilati C., Barros S.S. \& Castilhos L.M.L. 1992. Senecio spp. poisoning in cattle in Southern Brazil. Vet. Hum. Toxicol. 34(3):241-246.

Beskow W.B. 1995. A study of the factors influencing the emergence and establishment of ragwort (Senecio jacobaea L.) seedlings in pastures. M.Sc.Thesis, Massey University, New Zealand. 116p.

Bull L.B. 1955. The histological evidence of liver damage from pyrrolizidine alkaloids: megalocytosis of the liver cells and inclusion globules. Aust. Vet. J. 31:33-40.

Castellani T.T., Caus C.A. \& Vieira S. 1999. Fenologia de uma comunidade de duna frontal no sul do Brasil. Acta Botanica Brasilica 13(1):99114.

Coombs E.M., Bedell T.E. \& McEvoy P.B. 1991. Tansy ragwort (Senecio jacobaea): importance, distribution, and control in Oregon, p.419-428. In: James L.F., Evans J.O., Ralphs M.H. \& Child R.D. (ed.) Noxious Range Weeds. Westview Press, San Francisco.

Dickinson J.O. 1980. Release of pyrrolizidine alkaloids into milk. Proc. West. Pharmacol. Soc. 23:377-379.

Driemeier D. \& Barros C.S.L. 1992. Intoxicação experimental por Senecio oxyphyllus (Compositae) em bovinos. Pesq. Vet. Bras. 12(1/2):33-42.

Driemeier D., Barros C.S.L. \& Pilati C. 1991. Seneciose em bovinos. Hora Vet., Porto Alegre, 59:23-30.

Gava A. \& Barros C.S.L. 1997. Senecio spp poisoning of horses in southern Brazil. Pesq. Vet. Bras. 17(1):36-40.

Girardi-Deiro A.M. \& Gonçalves J.O.N. 1985. Estrutura da vegetação de um campo natural submetido a três cargas animais na região sudoeste do Rio Grande do Sul. Bolm Pesq. 1, Embrapa, Bagé, RS. 55p.

Girardi-Deiro A.M., Gonçalves J.O.N. \& Gonzaga S.S. 1992. Campos naturais ocorrentes nos diferentes tipos de solos no município de Bagé, RS. 2. Fisionomia e composição florística. Iheringia, Sér. Bot. 42:55-79.

Gonçalves J.O.N. \& Girardi-Deiro A.M. 1986. Efeito de três cargas animais sobre a vegetação de pastagem natural. Pesq. Agropec. Bras. 21(5):547554 .

Gonçalves J.O.N., Girardi-Deiro A.M. \& Gonzaga S.S. 1988. Campos naturais ocorrentes nos diferentes tipos de solos no município de Bagé, RS. 1. Caracterização, localização e principais componentes da vegetação. Bolm Pesq. 12, Embrapa, Bagé, RS. 28p.

Harper J.L. \& Wood W.A. 1957. Biological flora of the British Isles: Senecio jacobaea L. J. Ecology 45:617-637.

Hirschmann G.S., Ferro E.A., Franco L., Recalde L. \& Theoduloz C. 1987. Pyrrolizidine alkaloids from Senecio brasiliensis populations. J. Natural Products 50(4):770-772.

Ilha M.R.S., Loretti A.P., Barros S.S. \& Barros C.S.L. 2001. Intoxicação espontânea por Senecio brasiliensis (Asteraceae) em ovinos no Rio Grande do Sul. Pesq. Vet. Bras. 21(3):123-138.

Johnson A.E. \& Smart R.A. 1983. Effects on cattle and their calves of tansy ragwort (Senecio jacobaea) fed in early gestation. Am. J. Vet. Res. 44(7):1215-1219. 
Jubb K.V.F., Kennedy P.C. \& Palmer N. 1992. Pathology of Domestic Animals. Vol. 2. 4th ed. Academic Press, London. 747p.

Karam F.S.C., Méndez, M.C., Jarenkow, J.A. \& Riet-Correa, F. 2002. Fenologia de quatro espécies tóxicas de Senecio (Asteraceae) na região Sul do Rio Grande do Sul. Pesq. Vet. Bras. 22(1):33-39.

Kingsbury J.M. 1964. Poisonous Plants of the United States and Canada. Prentice-Hall, Englewood Cliffs, New Jersey. 626p.

Krebs H.C., Carl T. \& Habermehl G.G. 1996. Pyrrolizidine alkaloid composition in six brazilian Senecio species. Phytochemistry 43(6):12271229.

Liddell J.R., Stermitz F.R. \& Barros C.S. 1992. Pyrrolizidine alkaloids from Senecio oxyphyllus, a Brazilian poisonous plant. Biochem. System. Ecology 20(4):393.

Macedo W. 1984. Levantamento de reconhecimento dos solos do Município de Bagé. Documentos 1, Embrapa-UEPAE de Bagé. 69p.

MacLachlan N.J. \& Cullen J.M. 1998. Fígado, sistema biliar e pâncreas exócrino, p.95-131. In: Carlton W.W.\& McGavin M.D. (ed.) Patologia Veterinária Especial de Thomson. 2 $2^{\underline{a}}$ ed. Artes Médicas, Porto Alegre.

Martin S.W., Meek A.H. \& Willeberg P. 1988. Veterinary Epidemiology. Principles and methods. State University Press, Ames, Iowa. 343p.

Mattocks A.R. 1967. Spectrophotometric determination of unsaturated pyrrolizidine alkaloids. Analytical Chemistry 39:443-446.

Mattocks A.R. 1968a. Spectrophotometric determination of unsaturated pyrrolizidine alkaloids-some improvements. Analytical Chemistry 40:1749-1750.

Mattocks A.R. 1968b. Toxicity of pyrrolizidine alkaloids. Nature 217:723728 .

McClements I., Courtney A.D. \& Malone F.E. 1998. Management and edaphic factors related with the incidence of marsh ragwort, p.40-44. In: Garland T. \& Barr A.C. (ed.) Toxic Plants and other Natural Toxicants. Biddles Ltd. Guildford and King's Lynn, Oxon.

Méndez M.C. 1993. Intoxicação por Senecio spp., p.43-57. In: Riet-Correa F., Méndez M.C. \& Schild A.L. (ed.) Intoxicações por Plantas e Micotoxicoses em Animais Domésticos. Editorial Agropecuaria Hemisferio Sur, Montevideo.

Méndez M.C. \& Riet-Correa F. 1993. Intoxication by Senecio tweediei in cattle in southern Brazil. Vet. Hum. Toxicol. 35(1):55.

Méndez M.C. \& Riet-Correa F. 2000. Plantas hepatotóxicas, p.9-29. In: Méndez M.C. \& Riet-Correa F. (ed.) Plantas Tóxicas e Micotoxicoses. Editora Universitária, Pelotas.

Méndez M.C., Riet-Correa F. \& Schild A.L. 1987. Intoxicação por Senecio spp (Compositae) em bovinos no Rio Grande do Sul. Pesq. Vet. Bras. $7(2): 51-56$.

Méndez M.C., Riet-Correa F., Schild A.L. \& Martz W. 1990. Intoxicação experimental por cinco espécies de Senecio em bovinos e aves. Pesq. Vet. Bras. 10(3/4):63-69.

Molyneux R.J. \& Roitman J.N. 1980. Specific detection of pyrrolizidine alkaloids on thin-layer chromatograms. J. Chromatography 195:412415.

Nunes Vieira G.V. 1967. Criação de Ovinos. $3^{\underline{a}}$ ed. Melhoramentos, São Paulo. 480p.

Osweiler G.D. 1998. Toxicologia Veterinária. Artes Médicas, Porto Alegre. $526 \mathrm{p}$.

Pearson E.G. 1993. Moléstias do sistema hepatobiliar, p.839-857. In: Smith B.P. (ed.) Tratado de Medicina Interna de Grandes Animais. Vol. 1. Manole, São Paulo.

Peterson J.E. \& Culvenor C.C.J. 1983. Hepatotoxic pyrrolizidine alkaloids, p.637-671. In: Keeler R.F. (ed.) Plant and Fungal Toxins (Handbook of Natural Toxins). Vol.1. Marcel Dekker, New York.

Radostits O.M., Gay C.C., Blood D.C. \& Hinchcliff K.W. 1999. Veterinary Medicine. 9th ed. W. B. Saunders, London. 1881p.

Riet-Correa F. \& Medeiros R.M.T. 2001. Intoxicações por plantas no Brasil e no Uruguai: importância econômica, controle e riscos para a saúde pública. Pesq. Vet. Bras. 21(1):38-42.

Rouquayrol M.Z. 1990. Estrutura epidemiológica, p.221-242. In: Epidemiologia \& Saúde. $3^{\underline{a}}$ ed. MEDSI, Rio de Janeiro.

Schild A.L., Riet-Correa F., Méndez M.C., Ferreira J.L.M. \& Brod C.S. 1989. Relatório de atividades do Laboratório Regional de Diagnóstico e doenças diagnosticadas no ano de 1988. Editora Universitária, Pelotas. 59p.

Schild A.L., Motta A.C., Riet-Correa F., Karam F.C. \& Grecco F.B. 2004. Photosensitivity in cattle in southern Brazil, p. 162-166. In: Acamovic T., Stewart C.S., Pennycott T.W. (ed.) Poisonings Plants and Related Toxins. $\mathrm{CAB}$ International, London.

Soares M.P., Riet-Correa F., Méndez M.C., Rosa F.G. \& Carreira E.G. 2000. Controle biológico de Senecio spp. com pastoreio de ovinos. Anais da II Reunión Argentina de Patologia Veterinaria, Faculdad de Ciencias Veterinarias, Universidad Nacional del Nordeste, Corrientes, Argentina, p.79. (Resumo)

Summers B.A., Cummings J.F. \& deLahunta A. 1995. Degenerative diseases of the central nervous system, p.208-350. In: Veterinary Neuropathology. Mosby, St. Louis.

Thompson K., Grime J.P. \& Mason G. 1977.Seed germination in response to diurnal fluctuations of temperature. Nature 267:147-149.

Tokarnia C.H. \& Döbereiner J. 1984. Intoxicação experimental por Senecio brasiliensis (Compositae) em bovinos. Pesq. Vet. Bras. 4(2):39-65.

Tokarnia C.H., Döbereiner J. \& Peixoto P.V. 2000. Plantas hepatotóxicas, p.80-110. In: Tokarnia C.H., Döbereiner J. \& Peixoto P.V. (ed.) Plantas Tóxicas do Brasil. Editora Helianthus, Rio de Janeiro. 310p. 